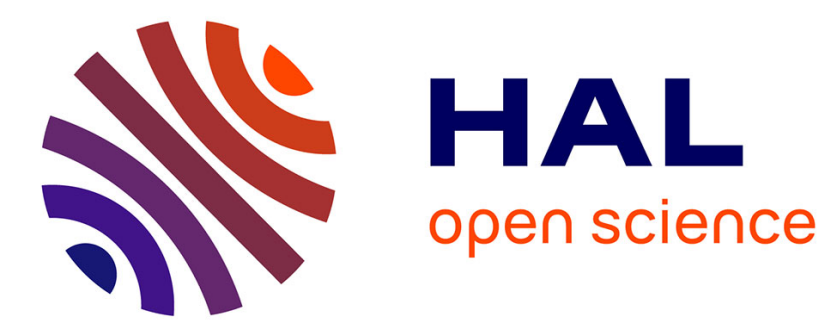

\title{
Electroosmosis near surfactant laden liquid-air interfaces
} Baptiste Blanc, Oriane Bonhomme, Pierre-Francois Brevet, Emmanuel Benichou, Christophe Ybert, Anne-Laure Biance

\section{To cite this version:}

Baptiste Blanc, Oriane Bonhomme, Pierre-Francois Brevet, Emmanuel Benichou, Christophe Ybert, et al.. Electroosmosis near surfactant laden liquid-air interfaces. Soft Matter, 2018, 14 (14), pp.2604 - 2609. 10.1039/C7SM02508D . hal-01909445

\section{HAL Id: hal-01909445 \\ https://hal.science/hal-01909445}

Submitted on 16 Feb 2022

HAL is a multi-disciplinary open access archive for the deposit and dissemination of scientific research documents, whether they are published or not. The documents may come from teaching and research institutions in France or abroad, or from public or private research centers.
L'archive ouverte pluridisciplinaire HAL, est destinée au dépôt et à la diffusion de documents scientifiques de niveau recherche, publiés ou non, émanant des établissements d'enseignement et de recherche français ou étrangers, des laboratoires publics ou privés. 


\title{
Electroosmosis near stress free surfaces at the molecular and macroscopic scales
}

\author{
Baptiste Blanc, Oriane Bonhomme, Pierre-Francois Brevet, \\ Emmanuel Benichou, Christophe Ybert, and Anne-Laure Biance* \\ Univ Lyon, Université Claude Bernard Lyon 1, CNRS, \\ Institut Lumière Matière, F-69622, VILLEURBANNE, France
}

\begin{abstract}
Generation of an electroosmostic (EO) flow near a liquid-gas interface covered with ionic surfactants is experimentally investigated. Combination of microscopic flow measurements with a molecular characterization of the interface by second harmonic generation (SHG) shows that, under an electrical forcing, the liquid underneath the surface flows while the adsorbed surfactants remain immobile. These unexpected results contribute to the fundamental understanding of electrokinetic phenomena in these complex situations. Besides, this new experimental technique opens the route to simultaneously probe the liquid flow near a soapy interface and the corresponding surfactant repartition affecting the hydrodynamic boundary condition.

PACS numbers: .
\end{abstract}

Effects of surface active materials on free surface flows have long been reported and are encountered in many applications involving liquid foams or sprays. Nowadays, liquid-gas materials with a controlled microstructure are used to design new technological systems for tissue engineering, optics, photonic crystals, and lab-on-a-chip applications [1]. These microstructured materials are usually produced using microfluidics techniques and overall require a fine control of liquid microflows near gas interfaces.

At these microscales, surface-driven flows - and among them surface-induced electrical flows - have become systematically used because of their integration capabilities and their favorable downsize scaling compared to classical bulk-driven flows [2]. However, unlike solidliquid interfaces, which have been investigated for more than two centuries [3], the understanding of electrokinetics near liquid-gas interfaces still raises numerous fundamental and practical questions.

In the vicinity of a charged surface, counter-ions are loosely attracted to the surface to form the so-called electrical Debye layer (EDL). When submitted to an electric field, the EDL experiences a net force and subsequent liquid motion then arises. This electro-osmotic flow (EO) propagates via viscous momentum diffusion into the neutral liquid bulk to eventually establish a plug flow profile at steady state. For solid surfaces, this plug-flow velocity $\boldsymbol{U}$ is given by the Smoluchovski relation [4]:

$$
\boldsymbol{U}=-\frac{\zeta \epsilon \boldsymbol{E}}{\eta}
$$

with $\epsilon$ and $\eta$ the liquid permittivity and viscosity, $\zeta$ the so-called $\zeta$-potential and $\boldsymbol{E}$ the applied electric field. The $\zeta$-potential does not only incorporate electrostatic properties of the interface but also accounts for the hydrodynamic boundary condition [4]. In particular, solid-liquid friction [5-8] and more generally charge mobility [9] have been shown to affect the EO response flow. In addition, the complex entanglement between surface electrostatic properties and nearby liquid dynamics at stake during
EO driving was furthermore illustrated by the experimental report of unexplained couplings as revealed by the non-linear optical response of surfaces [10].

In this respect, the case of liquid-gas interfaces, among which soapy interfaces, a priori incorporates an even richer phenomenology [11] due to the need to additionally consider the friction of the liquid on the surfactant layer [12] or the intrinsic rheology of the interface [13]. Depending on the surfactants used, the surface dynamics - and thus the relevant hydrodynamic boundary condition - can be tuned by orders of magnitude as shown for instance by drainage experiments in soap films $[14,15]$. Moreover, surfactant mobility can induce a redistribution of molecules that results in inhomogeneous surface concentrations and subsequent surface tension gradients. These so-called Marangoni stresses need to be considered to account for many experimental situations involving liquid dynamics near soapy interfaces [16-19].

Direct experimental probing of surfactant repartition in these situations has never been achieved so far. Then, we propose to investigate it in the case of electrically driven flow in the vicinity of surfactant-laden liquid-gas interfaces. In practice, we perform optical microflow velocimetry for quantifying surface EO response properties and the associated $\zeta$-potential, and second harmonic generation (SHG) experiments to locally quantify the surfactant surface concentration. These two measurements allow us to probe the surfactant repartition along the interface and to discuss how the complex hydrodynamic condition affects the underneath flow.

Firstly the investigation of soapy interfaces EO response, and the determination of their $\zeta$-potential were performed by using the experimental setup sketched in Fig. 1. It relies on measuring the surface-driven oscillatory (Stokes) boundary layer resulting from an AC electric field driving. Such an approach was proposed and validated to capture the electrokinetic response of solid surfaces [20].

The experimental cell, filled with the surfactant solu- 


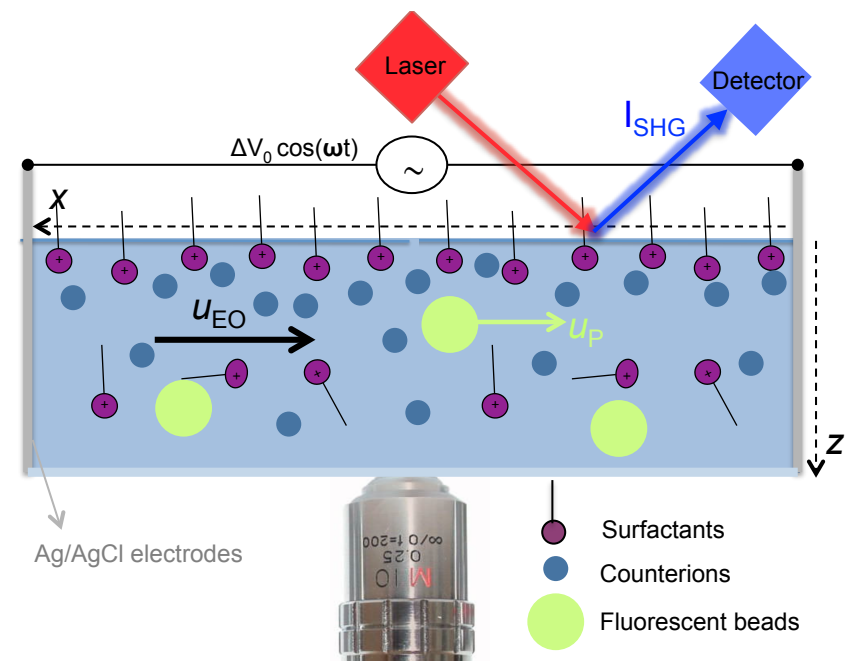

FIG. 1. Sketch of the experimental set-up (scales are not respected). $u_{E O}$ and $u_{P}$ correspond to the electroosmostic velocity of the liquid and the intrinsic electrophoretic velocity of the colloidal probes. The tracer displacements are recorded along the $z$ direction whereas the SHG intensity is scanned along the $x$ direction.

tion, is molded in PDMS (Polydimethylsiloxane). The liquid-filled volume is $1 \mathrm{~cm} \mathrm{x} 1 \mathrm{~cm}$ surface, $1 \mathrm{~cm}$ in depth and the cell bottom is sealed by an optical glass coverslip. Surfactant solutions are made of tetradecyltrimethylammonium bromide (TTAB) at concentrations $c$ ranging from $\mathrm{cmc} / 2000$ to $3 \mathrm{cmc}$, where cmc stands for the critical micellar concentration $(\mathrm{cmc}=4.5 \mathrm{mM}[21,22])$. A small amount of green fluorescent polystyrene spheres $(200 \mathrm{~nm})$ are added to the solution $\left(c_{s}=2 \cdot 10^{-3} \%[\mathrm{v} / \mathrm{v}]\right)$ as tracers for microflow velocimetry.

The electric field is imposed via two immersed $\mathrm{Ag} / \mathrm{AgCl}$ electrodes plates perpendicular to the interface (Fig.1) connected to a voltage generator (5 to 30V) delivering a sinusoidal signal $(\omega / 2 \pi=2 \mathrm{~Hz})$. AC driving is advantageously used to minimize electrode artifacts and to remove spurious drifts by extracting the - oscillatinglinear response contribution (see Supplemental Materials (SM) [23]). Fluorescent probe displacements are recorded for various distance $z$ from the liquid-gas interface with a high speed confocal microscope (Leica TCS SP5 DMI6000) and filtered at the driving frequency to isolate the linear response component at $\cos \omega t$.

The colloidal probes being charged particles, their observed oscillating velocity results from the superimposition of the advective EO flow under investigation and of the own electrophoretic motion of the particles. The latter writes $u_{P}(t)=U_{P} \cos \left(\omega t-\phi_{P}\right)$, with $U_{P}$ the electrophoretic velocity of the particles and $\phi_{P}$ a phase shift arising from the experimental probing method, and due to a finite experimental depth of field (SM [23]). As for the investigated EO flow, it is generated at the liquid-gas interface and diffuses in the liquid by viscosity. The resulting flow profile $u_{E O}(z)$ is equivalent to the one from

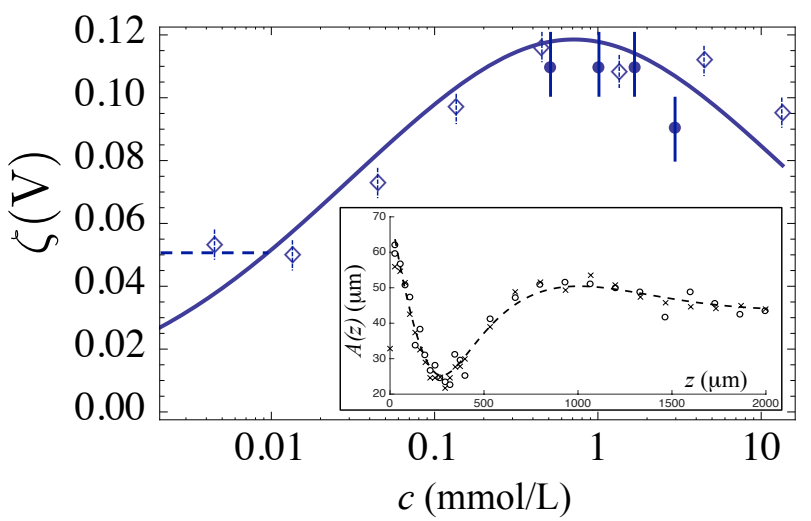

FIG. 2. Liquid-gas interface $\zeta$-potential vs surfactant bulk concentration $c .(\diamond)$ : Present measurements; $(-)$ : Prediction from SHG measurement and Grahame relation eq. (3) where $\Gamma$ is replaced by $\theta \Gamma$ with $\theta=0.076$ a fitting parameter corresponding to finite ionized ratio (see text); (--) Saturation plateau at $50 \mathrm{mV}$ (see text); (•): Surface potential measured by the thin film balance technique, data from [22]. Inset: Amplitude displacement depth profile $A(z)(c=0.135 \mathrm{mM}) .(\times$, $\circ)$ : data points recorded when scanning in $z$ back and forth respectively; (--): Theoretical fit according to eq. (2) with $U_{i}=5.0 \mu \mathrm{m} / \mathrm{s}, U_{P}=1.7 \mu \mathrm{m} / \mathrm{s}$ and $\phi_{P}=0.25$.

the Stokes oscillating boundary layer [24], $u_{E O}(z)=$ $U_{i} \exp \left(-z / z_{0}\right) \cos \left(\omega t-z / z_{0}\right)$ with $U_{i}=-\zeta \epsilon E / \eta$ the EO velocity in the vicinity of the surfactant layer and $z_{0}=\sqrt{2 \eta /(\rho \omega)}$ the length scale for viscous momentum diffusion.

Overall, the measured particle velocity $u_{t}(z)$ gathers both contributions so that the bead displacement amplitude $A(z)$ writes

$$
A(z)=\frac{U_{i}}{\omega} \sqrt{e^{-\frac{2 z}{z_{0}}}+2 \frac{U_{P}}{U_{i}} e^{-\frac{z}{z_{0}}} \cos \left(\frac{z}{z_{0}}-\phi_{P}\right)+\frac{U_{P}^{2}}{U_{i}^{2}}} .
$$

A typical measurement of $A(z)$ is reported in Fig. 2 (inset), together with the theoretical fit according to Eq.2, with $U_{P}, U_{i}$ and $\phi_{P}$ as free parameters.

Fig. 2 presents the soapy liquid-gas interface $\zeta$ potential deduced from the values of $U_{i}$ as a function of the surfactant bulk concentration $c$. The experimental curve $\zeta(c)$ displays several specific features: a nonmonotonous evolution with a maximum in $\zeta$ at intermediate $c$ and a saturation at the lowest concentration suggesting a finite $\zeta$ for vanishing concentrations. Remarkably, such a shape is in agreement with recent numerical simulations [12].

Because surface dynamics and (electro-)static properties are fundamentally entangled in the EO response as defined by $\zeta$, we now experimentally investigate the surfactant concentration at the liquid-gas interface. To that aim, we perform SHG measurements [25], a non-linear optical method, at the interface of the surfactant solutions. Indeed, SHG, whereby two photons at a fundamental frequency are annihilated and one at the harmonic frequency is generated, is a tool of choice to study interfaces. 


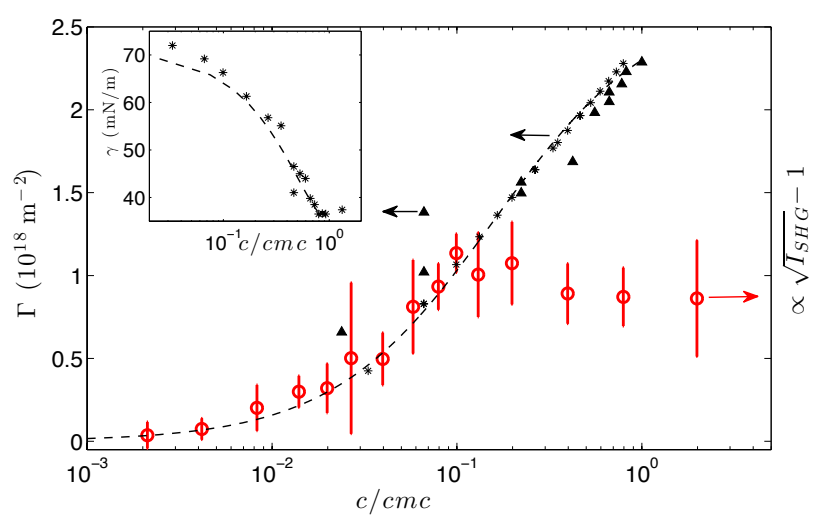

FIG. 3. Surfactant surface concentration $\Gamma$ vs surfactant bulk concentration $c /$ cmc. (०): SHG measurements based on $\sqrt{I_{S H G}}$ (see text); (*) Experimental surface tension $\gamma(c)$ (see inset) and Gibbs' law; $(\triangle)$ : Neutron reflectivity data from [27, 28]; (--) Langmuir isotherm fit of surface tension $\Gamma=a K c /(1+K c)\left(a=26.6410^{17} \mathrm{~m}^{-2}\right.$ and $K=1.404$ $\mathrm{L} / \mathrm{mmol})$. Inset: Surface tension $\gamma$ vs surfactant bulk concentration $c / c m c$. Symbols and lines: same as main figure.

As a matter of fact, this non-linear process is forbidden in centrosymetrical medium, such as air and liquid water so that only interfaces are probed and contribute to the observed signal. Experimentally, a femtosecond laser (wavelength $810 \mathrm{~nm}$ ) is focused at the liquid-air interface and the SHG intensity $I_{S H G}$ is collected in reflexion at the wavelength $405 \mathrm{~nm}$ by a detector composed of a spectrometer and a CCD camera [26]. Due to geometrical constraints, SHG measurements are thus performed in a larger experimental cell of upper free surface $4 \mathrm{~cm} \mathrm{x}$ $4 \mathrm{~cm}$. Obtained results have been checked to be identical for the original - smaller - cell size (SM [23]).

For adsorbed layer, it is commonly assumed that $\sqrt{I_{S H G}} \propto \Gamma\langle\beta\rangle$ with $\Gamma$ the surface concentration of the adsorbed molecules and $\langle\beta\rangle$ their hyperpolarisability, where brackets indicate orientational average [29]. Using the input and output $p$-polarisation SHG intensity (see SM [23]), the measured surface concentration is reported in Fig. 3 for over three decades in surfactant bulk concentration. Note that we checked by polarisation studies that the average molecular orientation does not change with surface concentration in good agreement with previous studies [30].

These SHG measurements can be compared to independent surface coverage parameters from surface tension measurements $\gamma(c)$ (inset of Fig. 3) assuming adsorbed species satisfy Gibb's law $\Gamma=-(1 / R T) \partial \gamma / \partial \ln (c)$ [31]. These data are very well fitted by a Langmuir isotherm $\Gamma=a K c /(1+K c)$, with $a=26.6410^{17} \mathrm{~m}^{-2}$ and $K=$ $1.404 \mathrm{~L} / \mathrm{mmol}$, in agreement with the literature [21]. Neutron reflectivity data for the same system [27, 28] also perfectly match the above estimates (Fig. 3). A very good agreement is obtained between SHG measurement (namely $\sqrt{I_{S H G}}$ ) and the previous surface cover- age values up to bulk concentrations of $0.1 \mathrm{cmc}$. We can note the extreme sensitivity of the SHG method to low surface coverage as it outperforms the two other experimental approaches by about a decade in bulk concentration. However, at higher concentrations, SHG signal no longer matches with the surface coverage. According to the above relationship between SHG intensity and surface coverage, this indicates a change in the hyperpolarisability factor $\langle\beta\rangle$. Indeed, $\langle\beta\rangle$ is affected by the local environment: the interactions between surfactants and ions due to surface charge saturation and subsequent ion binding effects $[12,21,22,32]$, the mean water molecule orientation [33] or an electric DC field induced bulk SHG response [34] can indeed contribute in this regime.

SHG proves extremely sensitive to probe the local and instantaneous surface concentrations of surfactant molecules, in the regime of low to moderate surface coverage, beyond which SHG response incorporates complex molecular environment contributions.

Thanks to this independent information on the surface coverage, we have clues concerning the electrostatic properties of the surfactant-laden interface, as surface charge can be assimilated at first stage as $e \Gamma$ with $e$ the elementary charge. We can now discuss in more details our $\zeta$-potential measurements of soapy interfaces, which results from the above charge properties and from the complex interfacial dynamics.

Indeed compared to usual charged solid surfaces, the surface charge (born by adsorbed surfactants) is a priori mobile, the friction of the liquid on the surface is either negligible or at least very low. In this respect, it is close to the problem of EO response of neutral hydrophobic surfaces investigated recently from a theoretical point of view $[9,35,36]$, where liquid-wall friction is reduced by slippage effects, and where ion specific adsorption is required to trigger an electrokinetic effect. Although the crucial role of charge mobility at the molecular scale (adsorbed species and counter ions) was put forward in this context, no experimental investigation has been proposed so far.

Assuming that the liquid friction with the surrounding air is negligible, the system \{adsorbed surfactants + water and ionic species $\}$ is isolated. Momentum conservation thus suggests that the bulk flow is compensated by a fast and opposite motion of adsorbed surfactants. If so, one would expect strong surface concentration gradients to build up at the scale of the trough in response to the electrical forcing.

In Fig. 4 we report the local (typical averaged over $1 \mathrm{~mm}$ ) and instantaneous (100 ms acquisition time for a $2 \mathrm{~Hz}$ forcing) SHG measurement scanned along the trough. No significant heterogeneities are measured suggesting that the surfactants remain evenly distributed within the technique accuracy. This shows that inhomogeneous surfactant repartition as schematized in Fig. 5(b) and subsequent Marangoni stress does not build up and that despite the EO movement of the liquid beneath, 
the interface remains stress free. In addition, let us mention that we do not report variations of the absolute SHG intensity with or without electric field, unlike some recent observations at solid-liquid interfaces [10]. The absence of surface concentration gradient may result from fast enough exchange with the bulk reservoir. However, additional experiments performed up to $100 \mathrm{~Hz}$ (for which surface/bulk surfactant exchanges are expected truly negligible) do not show any differences (see SM [23]).

These different observations then dismiss the possibility of strong motion of the adsorbed surfactant layer. This suggests that although very small, the frictional coupling between the liquid phase and the surrounding air cannot be ignored and act as a momentum reservoir. At steady-state, because the \{adsorbed surfactants + water and ionic species $\}$ system is globally neutral, no net driving force is exerted (Fig. 5a) so that no dissipative force can persist. However, air friction stress is acting on the system during a transient, to transfer momentum, and then to impose that the surfactants are motionless [36] before vanishing. Note that we may estimate whether a quasi steady state with zero outer stress can be achieved within the time scale of the forcing. During the transient regime, stress balance would read $\Gamma m \partial U_{s} / \partial t \sim \eta_{a} U_{s} / \sqrt{\nu_{a} t}$, with $m$ the mass of one surfactant, $U_{s}$ the surfactant velocity and $\eta_{a}$ and $\nu_{a}$ respectively the dynamic and kinematic viscosities of air. This results in a characteristic timescale given by $\Gamma^{2} m^{2} \nu_{a} / \eta_{a}^{2}$, of the order of $2 \mu \mathrm{s}$ (with $\Gamma=10^{19} \mathrm{~m}^{-2}, \mathrm{~m} \simeq 6.10^{-25} \mathrm{~kg}, \eta_{a}$ $=1.810^{-5} \mathrm{~Pa}$.s and $\nu_{a}=1.610^{-5} \mathrm{~m}^{2} / \mathrm{s}$ ), then far below the timescale of our experiments. Same reasoning can be performed with different sources of dissipation such as the friction of the surfactants on the lateral borders of the tank.

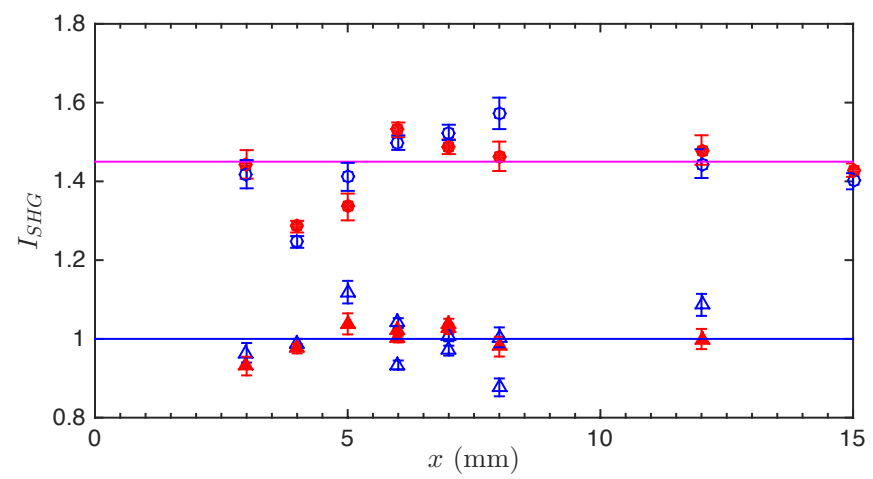

FIG. 4. SHG intensity at the liquid-gas interface for different position $x$ between the electrode. ( $\circ, \bullet)$ TTAB solution at $0.02 \mathrm{cmc}$ at respectively $(100 \mathrm{~V} / \mathrm{m}, 2 \mathrm{~Hz})$ electric field driving and without external driving; $(\Delta, \boldsymbol{\Delta})$ same for pure water subphase and interface.

Knowing that, unexpectedly, a zero velocity condition applies at the interface, we can identify the $\zeta$-potential with the surface potential of the interface $V_{s}$ deduced
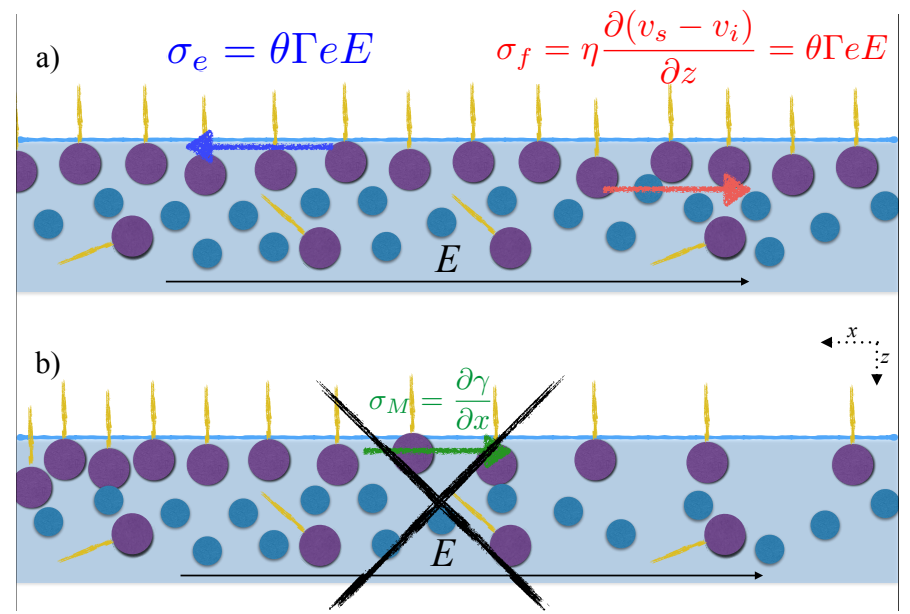

FIG. 5. (a)-Sketch showing the stress balance at the water/air interface in presence of surfactants and of an electric field $\boldsymbol{E}$. (b)-Sketch showing the scenario of an inhomogeneous surfactant repartition inducing a Marangoni stress at the interface.

from surfactant coverage $\Gamma$ by using the non-linear Poisson Boltzmann approximation. We assume that the surface charge is $\Gamma_{e}=\theta e \Gamma$ with $\theta$ a coefficient accounting for the surfactant partial dissociation or counter-ion binding effect as already reported in the literature for TTAB surfactants among others [12, 21, 32, 37-40], and then, if we use the Grahame relation [37], surface potential writes

$$
V_{s}=\frac{2 k T}{e} \sinh ^{-1}\left(\theta e \Gamma \sqrt{\frac{\pi}{2 k_{B} T \epsilon \mathcal{N}_{A} c}}\right)
$$

with $\mathcal{N}_{A}$ the Avogadro number. The value of $\Gamma$ is evaluated by the fitted Langmuir isotherm (see Fig. 3). As can be seen in Fig. 2, the above calculation for $V_{s}$ closely follows the evolution $\zeta(c)$, in particular with the appearance of a maximum close to $\mathrm{cmc} / 10$. Beyond the general shape, the quantitative agreement is obtained with a value $\theta=7.6 \%$ for the surfactant ionization ratio. A detailed investigation of this ion binding effect is beyond the scope of the present article, involving beyond meanfield Poisson-Boltzmann approaches [41, 42]. However, interestingly, such a low and constant ionization ratio is in quantitative agreement with static measurements of surface potential in similar systems [21, 22].

In addition to the above analysis, it has been recently put forward that despite the surfactant immobility, some dynamical effects may be present especially at low concentration, in the form of slippage between surfactant heads and the underneath liquid phase [12]. In this case, enhancement by a factor $b / \lambda$ is expected $[5,6], b$ being the slip length at the interface [4] and $\lambda$ the Debye length $(\lambda=\sqrt{\epsilon k T / 2 e c})$. In recent numerical simulations [12], slip lengths were predicted to be inversely proportional to the water friction on the surfactant heads and then to read $b=1 /(3 \pi R \Gamma)$, with $R$ the size of the surfactant head (around $0.5 \mathrm{~nm}$ for TTAB). Interestingly, this effect predicts that in the limit of low bulk and surface concen- 
tration, the $\zeta$-potential saturates at a value of $e / 3 \pi \epsilon R$ [12], around $50 \mathrm{mV}$ for TTAB in very good agreement with the experimental value reported at low concentration, as observed in Fig. 2.

Overall, we have investigated here the electrokinetic response of surfactant-laden interfaces by a combination of EO response measurements by microflow velocimetry and surface concentration distribution by SHG technique. Unlike what has been proposed for other actuations, e.g. in soap film drainage, our data are consistent with a motionless and stress-free surfactant layer, and allow a first analysis of the complex interplay between the adsorbed layer static and dynamic properties. In a wider perspective, the development of this new experimental technique on surfactant laden interfaces opens the way of probing surfactant repartition for different types of flow forcing like thermal forcing [43] or pressure driven, where Marangoni stress is expected [44]. It would be particularly interesting to test the case where the $\zeta$-potential is evaluated through streaming current measurements to see if the reciprocity relationship holds in such a complex situation.

\section{ACKNOWLEDGMENTS}

The authors would like to thank L. Joly and F. Detcheverry for fruitful discussions, R. Fulcrand and G. Simon for device realization. This project has been funded by ANR through EFOAM project ANR-13-JS090002 .

* anne-laure.biance@univ-lyon1.fr

[1] A. Huerre, V. Miralles, and M.-C. Jullien, Soft Matter 10, 6888 (2014).

[2] L. Bocquet and P. Tabeling, Lab on a Chip 14, 3143 (2014).

[3] F. F. Reuss, Notice sur un nouvel effect de l'électricité galvanique, Vol. 2 (Mémoire Soc. Imp. des Naturalistes de Moscou, 1809) pp. 327-336.

[4] L. Bocquet and E. Charlaix, Chemical Society Reviews 39, 1073 (2010).

[5] V. M. Muller, I. P. Sergeeva, V. D. Sobolev, and N. V. Churaev, Colloid Journal Of The Ussr 48, 606 (1986).

[6] L. Joly, C. Ybert, E. Trizac, and L. Bocquet, Phys. Rev. Lett. 93, 257805 (2004).

[7] C. I. Bouzigues, P. Tabeling, and L. Bocquet, Phys. Rev. Lett. 101, 114503 (2008).

[8] M. Audry, A. Piednoir, P. Joseph, and E. Charlaix, Faraday Discussion 146, 113 (2010).

[9] S.R. Maduar, A.V. Belyaev, V. Lobaskin, and O.I. Vinogradova, Phys. Rev. Lett. 114, 118301 (2015).

[10] D. Lis, E. H. G. Backus, J. Hunger, S. H. Parekh, and M. Bonn, Science 344, 1138 (2014).

[11] O. Bonhomme, O. Liot, A.-L. Biance, and L. Bocquet, Phys. Rev. Lett. 110, 054502 (2013).
[12] L. Joly, F. Detcheverry, and A.-L. Biance, Phys. Rev. Lett. 113, 088301 (2014).

[13] D. Langevin, Annual Review of Fluid Mechanics 46, 47 (2014).

[14] I. Cantat, S. Cohen-Addad, F. Elias, F. Graner, R. Hohler, O. Pitois, F. Rouyer, and A. Saint-Jalmes, Les mousses : Structure et dynamique, edited by Belin (Echelles, 2010).

[15] S. A. Koehler, S. Hilgenfeldt, E. R. Weeks, and H. A. Stone, Phys. Rev. E 66, 040601 (2002).

[16] C. Ybert and J.-M. Di Meglio, The European Physical Journal E 3, 143 (2000).

[17] P.-G. de Gennes, Langmuir 17, 2416 (2001).

[18] N. Denkov, S. Tcholakova, K. Golemanov, K. Ananthapadmanabhan, and A. Lips, Phys. Rev. Lett. 100, 138301 (2008).

[19] V. Miralles, E. Rio, I. Cantat, and M.-C. Jullien, Soft Matter 12, 7056 (2016).

[20] J. C. Corbett, F. McNeil-Watson, R. O. Jack, and M. Howarth, Colloids and Surfaces A: Physicochemical and Engineering Aspects 396, 169 (2012).

[21] V. Bergeron, Langmuir 13, 3474 (1997).

[22] J. Schulze-Schlarmann, N. Buchavzov, and C. Stubenrauch, Soft Matter 2, 584 (2006).

[23] B. Blanc, O. Bonhomme, P.-F. Brevet, E. Benichou, C. Ybert, A.-L. Biance, "Supplemental Information," http://.

[24] P. Dutta and A. Beskok, Analytical Chemistry 73, 5097 (2001).

[25] Y. Shen, Nature 337, 519 (1989).

[26] G. Martin-Gassin, E. Benichou, G. Bachelier, I. RussierAntoine, C. Jonin, and P.-F. Brevet, The Journal of Physical Chemistry C 112, 12958 (2008).

[27] C. Stubenrauch, V. B. Fainerman, E. V. Aksenenko, and R. Miller, J. Phys. Chem. B 109, 1505 (2005).

[28] E. A. Simister, E. M. Lee, R. K. Thomas, and J. Penfold, The Journal of Physical Chemistry 96, 1373 (1992).

[29] K. Eisenthal, Chemical Reviews 96, 1343 (1996).

[30] C. M. Johnson and E. Tyrode, Physical Chemistry Chemical Physics 7, 2635 (2005).

[31] B. Cabane and S. Hénon, Liquides: solutions, dispersions, émulsions, gels (Belin Paris, 2003).

[32] C. M. Phan, J. Phys. Chem. B 120, 7681 (2016).

[33] K. T. Nguyen, A. V. Nguyen, and G. M. Evans, J. Phys. Chem. C 119, 15477 (2015).

[34] Y.-C. Wen, S. Zha, X. Liu, S. Yang, P. Guo, G. Shi, H. Fang, Y. R. Shen, and C. Tian, Phys. Rev. Lett. 116, 016101 (2016).

[35] D. M. Huang, C. Cottin-Bizonne, C. Ybert, and L. Bocquet, Phys. Rev. Lett. 98, 177801 (2007).

[36] D. M. Huang, C. Cottin-Bizonne, C. Ybert, and L. Bocquet, Langmuir 24, 1442 (2008).

[37] J. Israelachvili, Intermolecular and Surface Forces, Third Edition (Academic Press, 2010).

[38] V. Kalinin and C. Radke, Colloids and Surfaces A: Physicochemical and Engineering Aspects 114, 337 (1996).

[39] R. Zana, S. Yiv, C. Strazielle, and P. Lianos, Journal of Colloid and Interface Science 80, 208 (1981).

[40] P. Carpena, J. Aguiar, P. Bernaola-Galván, and C. Carnero Ruiz, Langmuir 18, 6054 (2002).

[41] D. Ben-Yaakov, D. Andelman, R. Podgornik, and D. Harries, Current Opinion in Colloid \& Interface Science 16, 542 (2011). 
[42] M. A. Brown, Z. Abbas, A. Kleibert, R. G. Green, A. Goel, S. May, and T. M. Squires, Physical Review X 6, 011007 (2016).
[43] V. Miralles, B. Selva, I. Cantat, and M.-C. Jullien, Phys. Rev. Lett. 112, 238302 (2014).

[44] C. J. W. Breward and P. D. Howell, Journal of Fluid Mechanics 458, 379 (2002) 